\title{
Pedagogía Social: UNA construcción para el aprendizaje de la organización de redes comunales socio-educativas. Un estudio cruzado entre las comunidades de Santa Rita y Los Chiles de la provincia de Alajuela
}

Social Pedagogy: A National University of Costa Rica Project About Constructing for Learning the Organization of Social-Educational Community Networks. A Cross-Study Between the Communities of Santa Rita and Los Chiles in the Province of Alajuela, Costa Rica

\author{
Isabel Badilla Zamora \\ Universidad Nacional \\ Heredia, Costa Rica \\ isabel.badilla.zamora@una.cr \\ Rafael Esteban Jiménez Corrales \\ Universidad Nacional \\ Heredia, Costa Rica \\ rafael.jimenez.corrales@una.cr \\ Heidy León Arce \\ Universidad Nacional \\ Heredia, Costa Rica. \\ heidy.leon.arce@una.cr \\ Silvia Segura Esquivel \\ Universidad Nacional \\ Heredia, Costa Rica \\ silvia.segura.esquivel@una.cr \\ Gabriela Solís Sánchez \\ Universidad Nacional \\ Heredia, Costa Rica \\ maria.solis.sanchez@una.cr
}

Recibido: 09/03/2018 Aceptado: 28/06/2018 
Revista Universidad en DiÁlogo • Vol. 8, N. ${ }^{\circ} 2$, Julio-Diciembre, 2018, pp. 99-124

ISSN 2215-2849 • EISSN: 2215-4752

DOI: http://dx.doi.org/10.15359/udre.8-2.7

Resumen. El proyecto Pedagogía Social: UNA construcción para el aprendizaje de la organización de redes comunales socio-educativas: Un estudio cruzado entre las comunidades de Santa Rita y Los Chiles de la provincia de Alajuela 2016-2018; ubicado en la División de Educación Básica (DEB, en el Centro de Investigación y Docencia en Educación (CIDE), de la Universidad Nacional (UNA); a través de la experiencia de campo propone en el presente escrito, el análisis de las estructuras organizacionales y de convivencia social, para la comprensión de factores que inhiben y potencian el desarrollo social de las comunidades, desde una lógica de entrecruzamiento socio-histórico y de esa forma, generar acciones propias de la educación social para la promoción de aprendizajes organizacionales en el desarrollo de redes comunales socio-educativas en dichas comunidades. Para alcanzar los objetivos se planean una serie de estrategias que se enmarcan tanto dentro del paradigma de la investigación interpretativa- naturalista; así como, dentro del paradigma de la investigación socio- crítica, donde la reflexión se convierte en parte fundamental, ya que, valora la formación y transformación de las poblaciones participantes. Se trabaja bajo el enfoque de entrecruzamientos históricos para identificar características comunes que se generan dentro de dinámicas mayores que de alguna manera ayuden a explicar las dinámicas sociales. Una de las conclusiones más relevantes, es comprender que las acciones socio-pedagógicas desarrolladas desde el proyecto, han permitido la creación de formas dialógicas democráticas, con el fin de vislumbrar las necesidades de las comunidades e insertarse en sus metas y expectativas; así como, valorar la recreación y la celebración comunal como formas de empoderamiento de la acción social, política y económica de dichas poblaciones.

Palabras claves: cambio social, educación social, pedagogía social; redes comunitarias; ocio y recreación; extensión, investigación cualitativa.

Abstract. Social Pedagogy: A National University of Costa Rica Project about constructing for learning the organization of social-educational community networks. A cross-study between the communities of Santa Rita and Los Chiles of the province of Alajuela, Costa Rica, 2016-2018, is a project run in the Department of Basic Education (DEB) at the Center for Research and Teaching in Education (CIDE) of the National University (UNA) of Costa Rica. Through the field experience collected with this project, this article proposes the analysis of organizational structures and of social coexistence to understand factors that inhibit and potentiate the social development of the communities from a logic of socio-historical intertwining and, in this way, generate actions of social education for the promotion of organizational learning in the development of social-educational community networks in these communities. To achieve the objectives, planned strategies are framed within the paradigm of interpretative-naturalist research, as well as within the paradigm of socio-critical research where reflection becomes a fundamental part, since it values 
the formation and transformation of the participating populations. We work with the approach of historical intersections to identify common characteristics that are generated within larger dynamics that in some way help to explain social dynamics. One of the most relevant conclusions is to understand that the socio-pedagogical actions developed from the project have allowed the creation of democratic dialogical forms, in order to discern the needs of the communities and enter into their goals and expectations, as well as to value the recreation and communal celebrations as forms of empowerment of the social, political and economic action of these populations.

Keywords: social change, social education, social pedagogy, community networks, leisure and recreation, extension, qualitative research.

\section{Introducción}

La División de Educación Básica (DEB) del Centro de Investigación y Docencia en Educación (CIDE) ha venido desarrollando en los últimos años proyectos de investigación, extensión y docencia que permiten contribuir con la concretización de los más altos valores de la sociedad costarricense que favorezcan a aquellas comunidades más vulnerables del país.

Como parte de su quehacer, un grupo de académicos y académicas desde el año 2012 formula el proyecto Pedagogía Social: UNA Pedagogía sin Paredes, el cual atendió indicadores en el tema de educación y salud. Con este proyecto se inicia el gran interés de trabajar en zonas vulnerables del país en la provincia de Alajuela, como San Rita (El Infiernillo) en el cantón Central y las comunidades de San Gerardo, El Cachito y Punta Cortés pertenecientes al cantón de Los Chiles (frontera norte), dichas poblaciones se caracterizan por el alto nivel de pobreza, desempleo, ruralidad, marginalidad y migración; este panorama se convierte en un desafío para el grupo de académicos y académicas. Se emprenden desde la formación académica del estudiantado de la DEB acciones en el campo de la extensión universitaria, lográndose una importante integración entre docencia, extensión e investigación.

A partir de esta iniciativa, se plantea el proyecto para el período 2016-2018 Pedagogía Social: UNA construcción para el aprendizaje de la organización de redes comunales socioeducativas. Un estudio cruzado entre las comunidades de Santa Rita y Los Chiles de la provincia de Alajuela, el cual apunta a identificar y valorar en las comunidades las formas organizacionales establecidas, como un elemento que enriquece o limita la calidad de vida de 
ReVista UniversidAd EN DiÁlogo • Vol. 8, N. ${ }^{\circ}$ 2, Julio-Diciembre, 2018, pp. 99-124

ISSN 2215-2849 • EISSN: 2215-4752

DOI: http://dx.doi.org/10.15359/udre.8-2.7

los grupos sociales, acoge grupos de poblaciones excluidas para favorecer la convivencia y promueve la igualdad y equidad social.

Desde esta perspectiva, el proyecto asume un compromiso importante en la búsqueda de vincular instancias y crear redes comunales socioeducativas en Santa Rita y Los Chiles de Alajuela. Un aspecto importante por señalar es que el proyecto se centra en valorar estructuras organizacionales y la convivencia social para comprender los factores que inhiben y potencian el desarrollo social de las comunidades, y generar acciones socioeducativas como respuesta a las necesidades que se presentan en estos poblados y a la vez nutrir con propuestas metodológicas en educación social los conocimientos del equipo de investigación.

Esta temática se vincula directamente con el plan de fortalecimiento de la DEB, el cual presenta como área emergente la pedagogía social. La cual se vincula con un marco amplio de análisis de la complejidad humana.

Con el desarrollo de los objetivos, tanto del proyecto como del presente artículo (tabla 1), se pretende favorecer, potenciar y diversificar las oportunidades socioeducativas de sectores vulnerables, marginados, en riesgo social. $\mathrm{Su}$ intención es propiciar el cambio para la construcción de una sociedad más justa y equitativa a través de la identificación, fortalecimiento de redes comunales y co-creación de estrategias que incentiven el ocio y la recreación.

Tabla 1

Objetivos de la investigación-extensión

\begin{tabular}{|l|}
\hline \multicolumn{1}{|c|}{ Objetivos } \\
\hline 1. Valorar las estructuras organizacionales y de convivencia social para la \\
comprensión de factores que inhiban y potencien el desarrollo social de las \\
comunidades de Santa Rita y Los Chiles de Alajuela, desde una lógica de \\
entrecruzamiento sociohistórico.
\end{tabular}

Fuente: elaboración propia a partir del proyecto, 2016. 
De esa forma, la DEB con el desarrollo de este proyecto logra cumplir con la misión y visión de la Universidad Nacional, acercándose a las comunidades vulnerables para de forma colectiva resolver necesidades e intereses compartidos. Visto así, la universidad no solo debe enfocarse en los problemas económicos de las comunidades, sino que debe ser un factor importante para promover el cambio social y desarrollo del entorno. Por lo tanto, se requiere una visión integral en la acción sustantiva que permita la creación y difusión del conocimiento entre lo que la sociedad demanda y lo que la universidad ofrece como institución de educación superior pública.

\section{Marco teórico}

\section{La pedagogía general frente a la pedagogía social}

En la actualidad, posmoderna, formada por imágenes, de poca profundidad, los términos y conceptos suelen volar como polvo en el viento, pedagogía, metodología, mediación, principios, suelen asumirse muchas veces de forma ligera, con base teórica pobre y con mínima reflexión.

En este apartado seabordan concepciones pedagógicas creadas porprofesionales en pedagogía general, se analiza cómo dichas conceptualizaciones y tendencias impactan en el campo de la pedagogía social y se establece un discurso que desde lo pedagógico permita adoptar una posición en el convulso mundo conceptual que ha rodeado y rodea a la educación.

En su texto Pedagogía del conocimiento, el pedagogo Flórez (2005) realiza una de las más completas ideas en torno al concepto de pedagogía, como la ciencia de la educación. Su planteamiento histórico-hermeneútico desemboca en una visión unitaria de pedagogía, que busca incorporar diferentes conceptos y tradiciones. Para Flórez (2005), la pedagogía es un todo unitario, una ciencia social que se estructura en tres niveles indivisibles y complementarios uno de otro. En el primer nivel, producto de la historia y de la cultura, se encuentran las corrientes pedagógicas, basadas en los principios pedagógicos que nacen de la tradición pedagógica, de la ética y de los aportes de las ciencias de la educación. ${ }^{1}$

Las ciencias de la educación son saberes o prácticas teórico-metodológicas que alumbran, ayudan y colaboran con la pedagogía. Su objeto de estudio no es el hecho educativo en la mayoría de los casos, pero pueden abordar a este desde su propia lógica. 
En el primer nivel se problematiza sobre la formación humana, centro neural de la acción educativa; dicho ideal varía en función de las corrientes pedagógicas. A ese nivel Flórez (2005) lo denomina como el nivel teórico-formal. Los principios pedagógicos son elementos constantes que se han repetido a lo largo del tiempo cuando se hace educación, conscientes o no, ellos se materializan en las prácticas de los procesos de enseñanza y aprendizaje.

Por su nivel de complejidad y por no encontrarse siempre explícitos suelen escapar de la acción educativa en aulas o espacios pedagógicos en general, pero la conciencia y reflexión de ellos y ante todo de la formación humana marcarán una gran diferencia entre un pedagogo o una pedagoga y una persona enseñante que aplica y genera técnicas. Esa aplicación acrítica e instrumentalizada puede llegar hasta la generación de procesos no educativos, aunque los mismos en apariencia sí lo parezcan. La conciencia de la formación humana mediante la educación es tarea de la persona pedagoga.

En la propuesta de Flórez (2005), la persona docente de aula o espacio pedagógico consciente de su acción, apropiada de la concepción de formación humana, va camino a constituirse en profesional de la pedagogía. ${ }^{2}$

Es importante destacar que dentro de los principios pedagógicos fundamentales, según Florez (2005), se ubican los siguientes:

- El afecto

- La experiencia

- El diseño o configuración del medio

- El desarrollo progresivo

- La actividad y su vínculo con el aprendizaje

- La actividad lúdica

- La función de la persona educadora

- El cogobierno o formas de relación entre personas involucradas

- La diferencia en el aprendizaje individual

- La actividad y las formas de asociación grupales

Se habla tanto de aula como de espacio pedagógico porque el proceso de aprendizaje no solo acontece en un aula, es más, en el caso de la educación social suele no ser por lo general en ella. 
Este autor establece un segundo nivel de conceptualización de la pedagogía: los modelos pedagógicos, los cuales son denominados como el "nivel teórico de intermediación y recontextualización” (Flórez, 2005. p. 123), caracterizado por su construcción desde la hermeneútica. Incluye una forma de mayor operacionalización de las corrientes pedagógicas, los modelos pedagógicos están compuestos por cinco dimensiones fundamentales, para efectos de análisis se incluye una sexta dimensión y se reconceptualiza una de ellas:

- Metas de formación. Relacionado con el tipo de ser humano y sociedad que se aspira formar.

- Vínculos. ${ }^{3}$ Se reconceptuliza al contemplar el escenario como conformado por las interacciones y sus formas concretas entre docentes-estudiantes, estudiantes-estudiantes, estudiantes-contenidos, docentes-contenidos y otras formas vinculares complejas.

- Métodos. Las estrategias y formas de abordar los saberes por parte de los y las docentes a nivel conceptual, procedimental y actitudinal

- Contenidos. Los saberes conceptuales, actitudinales y procedimentales que se privilegian socialmente.

- Desarrollo. Se relaciona con la concepción de aprendizaje y cómo se genera este en los seres humanos.

- Valoración. Posiblemente se podría incluir en los métodos. Esta dimensión se incorpora en la medida en que la evaluación se consolida como un campo con un amplio bagaje teórico-metodológico que ha existido a lo largo de muchos años.

Flórez (2005) propone un tercer nivel o dimensión del constructo pedagogía, el cual está constituido por la realidad del aula o espacio pedagógico, el encuentro pedagógico donde se genera en el tiempo y el espacio el hecho educativo. En dicho encuentro aparecen tres actores esenciales, la persona docente, mediadora, instructora, según sea el modelo que ilumina la función del actor. El alumno o la alumna, aprendiente, estudiante, participante y un contenido que busca enseñarse y aprenderse. Como se insinuó, las características, funciones y nomenclatura de cada componente vendrán condicionadas de

En la conceptualización de Florez (2005) se reduce a la relación docente-alumnos/as. 
ReVista UniversidAd EN DiÁlogo • Vol. 8, N. ${ }^{\circ}$ 2, Julio-Diciembre, 2018, pp. 99-124

ISSN 2215-2849 • EISSN: 2215-4752

DOI: http://dx.doi.org/10.15359/udre.8-2.7

alguna manera por el modelo pedagógico que ilumine dicho hecho educativo y por la propuesta curricular que se haya elegido y estructurado.

La pedagogía social, siendo una ciencia relativamente joven, en cuanto pedagogía es importante mirarla desde el marco conceptual propuesto anteriormente, en el cual toda actividad científica a la que se le atribuya el nombre de pedagogía merece ser pensada desde los niveles y reflexiones presentes en esta concepción unitaria.

Por otra parte, se considera que el discurso teórico de la pedagogía social no puede ser propiedad única de los contextos europeos y del Primer Mundo. América Latina posee una larga y fructífera producción y reflexión sobre uno de los elementos fundamentales de la pedagogía social, la acción educativa para sectores tradicionalmente excluidos social, económica y políticamente hablando.

Inicialmente se analizará la idea de formación. Al igual que la pedagogía general, la pedagogía social tiene como elemento fundamental la formación humana. Persigue el desarrollo, la humanización. Creemos que esa humanización posee un carácter colectivo. La pedagogía social busca la humanización colectiva, el reconocimiento histórico de grupos que de alguna forma han sido y son excluidos, llámese población infantil en la calle, personas adultas mayores o sectores campesinos desplazados de su actividad agríco1a. La pedagogía social persigue una formación que no se contenta o conforma con el crecimiento y desarrollo pleno de una persona, apunta al grupo, al colectivo. Desde la propuesta de los autores se observa ese colectivo desde las tres dimensiones que propone Morin (2014), colectivos que son de forma compleja entendidos como sociedad, individuos y especie.

Otro elemento que se desea analizar está vinculado con los principios pedagógicos de la pedagogía social. Flórez (2005) propone diez grandes principios para la pedagogía general, a continuación se pretende brindar una mirada de ellos desde la acción de la pedagogía social.

- El afecto: Toda acción educativa social necesita partir de un afecto. Una relación amorosa, en cuanto legítima de la comunidad o grupo social que intenta acompañar en su desarrollo. La comunidad es una red compleja, pero el pedagogo o la pedagoga social se empatiza con ese colectivo. No lo mira como objeto frío de estudio, lo comprende, lo valora y lo aprecia. 
- La experiencia: Si bien la experiencia individual no se diluye en una mirada desde la pedagogía social, cobra fuerte relevancia la experiencia colectiva. Focaliza su mirada en las construcciones que el grupo aprendiente ha tenido a lo largo de su experiencia histórica, de ahí que valora su herencia, evita violentar esa experiencia, extrae de ella los elementos que potencian al grupo, no suprime la historia, valora y revalora la historia colectiva que a su vez guarda un componente individual.

- El diseño o configuración del medio: Toda persona pedagoga piensa en el medio ambiente, analiza el medio en el cual se genera el hecho educativo y prepara un medio propicio para el mismo. Para el pedagogo o la pedagoga social es de vital importancia el medio. La educación se genera en medios diversos que deben ser analizados y pensados. Pueden darse en la calle, un parque, un campo abierto, una pulpería, el medio es fundamental. Y a su vez ese medio es preparado, pensado. En la pedagogía social el medio rompe con la norma y la estructuración formal, aparece así el café, la tertulia, la conversación, la reunión, la producción pictórica, la palabra de la comunidad.

- El desarrollo progresivo: Las comunidades o grupos sociales no son lo que se desea que sean, son lo que la experiencia histórica ha venido configurando. Para el pedagogo o la pedagoga social, los grupos no son estáticos, poseen dinámicas internas, fases evolutivas, crecimientos, bucles recursivos. Los grupos no son lineales, pero cambian, buscan de una u otra forma un acople con el medio, buscan la vida y no la muerte. Para ello hay que comprender su lógica de desarrollo, sus ritmos, sus etapas, sus transiciones.

- La actividad y su vínculo con el aprendizaje: La pedagogía social considera que los grupos aprenden ante todo en el hacer, en el vivir, en el actuar. Un grupo sin actividad es un grupo necrófilo y por tanto antieducativo. A partir de la experiencia en los contextos que se han trabajado, la actividad es un tanto más compleja porque para la acción social se requiere incluir el concepto de praxis tal y como lo desarrolló Freire (1985). Los grupos sociales ante todo accionan, se les debe proponer los momentos reflexivos sobre su accionar, para luego retornar con mayor fortaleza y claridad a la acción transformadora. Los grupos sociales aprenden en el hacer, se fortalecen en la reflexión y se transforman en la acción consciente. 
- La actividad lúdica: Podría pensarse ligeramente que la actividad lúdica es un elemento propio de la pedagogía para niños y niñas o bien para ambientes escolarizados. Pero las comunidades y grupos sociales poseen una lúdica, la requieren. El juego por lo general va acompañado del gozo y este genera procesos bioquímicos a nivel cerebral que facilitan el aprendizaje. Además, los grupos cobran empatía, generan confianza, inician procesos dialógicos cuando media el juego, la alegría, la incertidumbre.

- La función del educador y de la educadora: El/la educador/a social debe ser un/a mediador/a. Un ser humano que se vincula con los grupos, que los asume con la conciencia clara de su necesidad de formación para el mejoramiento social. Respeta la cultura en la que trabaja, no la violenta. Mira las ideas, piensa en que las creaciones colectivas de grupos excluidos no son siempre negativas porque no siguen los patrones o normas de la cultura de la que proviene el/la educador/a social. Genera oportunidades, crea andamiajes, genera espacios de pensamiento, crea propuestas conjuntas para el mejoramiento del grupo en el cual educa y se educa.

- El cogobierno o formas de relación entre personas involucradas: En la mirada de la pedagogía social debe imperar el cogobierno. Si bien el mismo representa a la vez un contenido de toda propuesta en zonas de exclusión y vulnerabilidad. Dichos grupos han nacido y se han desarrollado desde el autoritarismo, que se genera tanto en las relaciones comerciales como en las lógicas institucionales, sean médicas, espirituales o escolares.

- La diferencia individual en el aprendizaje individual: Uno de los elementos o principios fundamentales en toda propuesta de pedagogía social es la idea de aprendizaje social. Pero en un marco de complejidad el norte debe ser la concepción de Morin (2014) en cuanto a que el ser aprendiente es ante todo una triada, conformada por individuos, sociedad y especie sapiens sapiens.

- La actividad y formas de asociación grupales: La pedagogía social sienta gran parte de su lógica metodológica en la investigación-acción-participativa. No lo hace por capricho, esta es 
inherente al aprendizaje. La comunidad aprende en la acción, los esquemas hipotético-deductivos tienden a fracasar si no existe una acción razonada. ${ }^{4}$

Es preciso indicar que en la concepción de Flórez (2005) priva la historicidad de la pedagogía, eso hace que la propuesta teórica, mirando sus tres niveles, no siempre encuentre en la práctica una coherencia sistemática. La situacionalidad, la historia y el entorno generarán encuentros y desencuentros entre los niveles o dimensiones de la pedagogía que plantea el autor colombiano, este razonamiento aplica para la educación y para la pedagogía social.

\section{Estructuras organizacionales}

Inicialmente el proyecto se basó en el concepto de estructuras organizacionales de las comunidades, obviamente dicho concepto se encuentra sumamente ligado con la teoría empresarial. El mismo hace alusión a la forma en que opera una comunidad determinada. Como señala Barone (2009), es importante considerar algunas dimensiones para el estudio organizacional, dentro de las que se destacan: valores culturales básicos, tipo de estructura organizacional (piramidal con pocos niveles, piramidal con múltiples niveles), redes funcionales, estructuras planas, situaciones de aplicación.

Este concepto es visto por investigadores/as-extensionistas desde un enfoque un tanto más sistémico, ya que una organización comunal, para lograr su equilibrio dinámico, tenderá a movilizarse o a estructurarse de diferentes formas según sea la situación que ingresa dentro del sistema. El nivel de autonomía y la necesidad de tolerancia (entre más tienda a una lógica sistémica concebida en red, mayores niveles de tolerancia e inclusión). La situación de estabilidad e inestabilidad del entorno (los entornos siempre tenderán a ser inestables, la estabilidad supone la muerte o extinción de la misma comunidad y de toda forma de organismo vivo). El nivel de experiencias organizacionales de las partes, las cuales juntas formarán un todo mayor que las primeras. Y la claridad del propósito, que se vincula con un plan que es consciente de la incertidumbre, pero que aprende a danzar con ella, no a temerle o a rechazarla.

$4 \quad$ La aseveración anterior aplica para el trabajo pedagógico, ya que la educación social suele utilizar complementariedad metodológica. 
ReVista UniversidAd EN DiÁlogo • Vol. 8, N. ${ }^{\circ}$ 2, Julio-Diciembre, 2018, pp. 99-124

ISSN 2215-2849 • EISSN: 2215-4752

DOI: http://dx.doi.org/10.15359/udre.8-2.7

\section{Aprendizajes organizacionales}

Si consideramos las organizaciones sociales como sistemas dinámicos, ellas poseen la capacidad de aprender. Y su principal lógica de aprendizaje será la capacidad de organizarse. Una perturbación en su medio generará de alguna forma una reorganización, la cual se incorpora como aprendizaje comunitario y a la vez se genera un aprendizaje en el grupo extensionista que implica otra organización sistémica.

La llegada de la persona extensionista, sin violar la lógica comunitaria, implica una fuerza que, de acuerdo con su magnitud, provoca un desequilibrio, y la comunidad aprende para su nuevo acoplamiento. Siguiendo la lógica de que el todo es mayor que las partes, el aprendizaje comunitario es complejo, profundo y dinamizador, va más allá del aprendizaje individual. Es lo que Gutiérrez (2006) relaciona con el aprendizaje rizomático, el cual es definido por el autor de la siguiente forma: "Es producto de un trabajo intelectual muy intenso de largas horas de reflexión y exploración. De poner en juego los cinco sentidos y la intuición. De interactuar con el propio grupo y los demás grupos" (p. 57). Además, agrega Gutiérrez, "de invertir energía sueños y pasión en un proyecto que en momentos puede parecer casi imposible" (p. 57). El aprendizaje comunitario, sea en procesos organizacionales u otros, posee un poder y una profundidad que, aunque parezcan lentos en el tiempo, se sitúan por encima de él.

\section{Convivencia social}

Originalmente la convivencia social emanaba de un concepto utilizado en la teoría de las relaciones internacionales, y se vinculaba con una coexistencia en un marco de tensión político-militar. Tal como lo indican Berns y Fitzduff (2007), para finales del siglo XX y principios del XXI el término se modificó de manera que actualmente se vincula con el reconocimiento recíproco de derechos y deberes del ser humano e involucra el principio de inclusión económica, social, política y cultural.

Es decir, el término transita de una relación de prevención de la amenaza a una relación de inclusión y respeto. La convivencia social implicaría el respeto, la igualdad de oportunidades y el derecho a la participación en la dinámica social de una comunidad. Un conglomerado social tendrá niveles de convivencia social en la medida en que se plasme en su devenir 
la cohesión social, la integración social y la inclusión social, de lo contrario se estaría en presencia de alteraciones de la convivencia comunitaria.

\section{Entrecruzamiento sociohistórico}

El término estudios cruzados proviene de la teoría histórica, señala Acuña (2014) que

La idea básica de este enfoque es la definición de un punto de intersección donde se cruzan eventos y procesos que afectan los elementos que han entrado en contacto. En este sentido, no se trata de determinar conexiones, sino de identificar procesos de producción de fenómenos históricos que son solamente inteligibles como resultado de encruces que trascienden espacios nacionales. El encruzamiento es una especie de yunque o crisol en el cual los fenómenos que por él pasan ya no son los mismos. Pero, además, el entrecruce desata procesos de reacción en cadena. El supuesto de independencia o de no resonancia de los objetos de la historia comparada no es aplicable en tales situaciones. El interés de la llamada historia cruzada es que permite estudiar el proceso de producción de fenómenos al interior de los espacios nacionales determinados por fuerzas que se originan fuera de ellos (pp. 7-8).

El concepto de historias cruzadas es totalmente aplicable a situaciones sociales y educativas actuales, e incluso comparte su diferencia con la historia comparada. La idea de historia cruzada se antepone a la de la historia comparada, se decanta en este nuevo enfoque. La educación y la pedagogía tuvieron en los años sesenta, setenta y ochenta toda una línea de investigación e incluso se incorporó Educación Comparada como materia en sus planes de estudio. Siendo los hechos educativos y los procesos educativos y sociales elementos potencialmente históricos, es válido seguir el curso y las ideas de un modelo de entrecruzamiento. Los procesos sociales y procesos educativos nacionales se entrecruzan, se originan con fuerzas que ingresan y están aparentemente fuera de ellos.

\section{Redes comunales socioeducativas}

El concepto de red comunal es tributario de otras ciencias, pero siendo un concepto aplicable a todo sistema vivo, se ha extendido a las ciencias sociales y por tanto a la educación social. Señala Capra (2017), "en el nuevo 
ReVista UniversidAd EN DiÁlogo • Vol. 8, N. ${ }^{\circ}$ 2, Julio-Diciembre, 2018, pp. 99-124

ISSN 2215-2849 • EISSN: 2215-4752

DOI: http://dx.doi.org/10.15359/udre.8-2.7

pensamiento sistémico, la metáfora del conocimiento como construcción queda remplazada por la de la red. Al percibir la realidad como una red de relaciones nuestras descripciones forman también una red interconectada de conceptos y modelos en la que no existen cimientos" (p. 59).

Este autor incluso supera el concepto de nodos que articulan la red y ve a estos como redes en sí mismos, por cuanto la realidad es un sistema en red, interconectado. La visión de la comunidad desde esta lógica lleva a cambiar la mirada, no se podría ver la escuela, el centro de salud, el campo, la empresa frutera como entes autónomos, son redes en redes. De ahí que la activación de una red comunal debe partir del conocimiento de las redes que de previo existen en ese espacio, para luego integrarse en esa compleja trama. Como la red es per se una red en la que circula información, las redes comunales socioeducativas parten de ese principio.

\section{Metodología}

El proyecto fundamenta su metodología en los aportes de la investigación interpretativa-naturalista y la investigación sociocrítica, las cuales poseen como esencia la reflexión sobre los elementos constitutivos de la pedagogía como ciencia. Como investigación pedagógica la acción se valora en la transformación y formación de los seres humanos participantes. Lo cual es apropiado para el trabajo comunitario y pedagógico que junto con técnicas etnográficas van generando aprendizajes en diferentes contextos.

El tipo de enfoque que desarrolla el proyecto es de historias cruzadas, se buscan puntos de intersección donde se cruzan eventos y procesos de organización que de una u otra forma entran en contacto, y contribuyen a tener una visión más holística de los fenómenos sociales. Desde el proyecto, se busca entrecruzar la organización y educación en las comunidades con procesos de migración y exclusión en cada uno de los grupos. ${ }^{5}$

La metodología se basa en el esquema presentado por Tobón (2001) para la investigación pedagógica, el mismo parte del establecimiento de tres puntos medulares, a saber: situación-problema, análisis teórico, construcción

La idea de investigaciones cruzadas nace en el marco de la teoría histórica, donde varios procesos son vistos o explicados desde marcos no solo microanalíticos, sino que intentan relaciones con macroprocesos sociales. 
metodológica-verificación en el campo. Según el mismo autor, la metodología cualitativa utilizada desde los estudios de entrecruzamiento permite a los investigadores y las investigadoras extensionistas mayor comprensión de las realidades sociales, las necesidades y los intereses en las comunidades.

Dicha metodología es conocida como TRIÁNGULO SP-T-V. Es importante resaltar que, para la construcción metodológica, el grupo de investigación participa de manera activa en la búsqueda de posibles soluciones construidas tomando en cuenta los aportes de la comunidad. La misma utiliza el enfoque cualitativo, ya que este "permite recuperar el contexto y la dimensión humana" (Bedmar y Añaños, 2007, p. 145); de la misma forma, explora, comprende y lee las realidades con las que se trabaja desde la complejidad. Además de que se pretende la transformación social y, en este caso específicamente, la transformación de poblaciones marginales.

A través de diferentes mecanismos que se desarrollan en la ruta metodológica se identifican varias necesidades, las cuales despliegan acciones específicas que pretenden mejorar las condiciones, permitiendo el autoaprendizaje que ha sido compartido para resignificarlas y así establecer el vínculo universidad-sociedad.

Para efectos de organización y clarificación se delimitaron varios espacios con la meta de iniciar de forma conjunta la conformación de una red-grupo estable y autosostenido:

1. Se hace un reconocimiento de las comunidades mediante la observación participante y no participante, visitando las comunidades en estudio, realizando entrevistas informales basadas en narraciones y dibujos inducidos, tertulias grupales, además de varios encuentros orientados a la promoción del ocio y la recreación. Se hace un diagnóstico participativo con líderes comunales y otros actores sociales, que permite identificar factores económicos, sociales, culturales, espaciales y geográficos, políticos y organizacionales

2. Seanaliza la información recolectada en las actividades anteriormente mencionadas, para ello se elaboran matrices sobre redes sociales por cada una de las comunidades. Se sistematizan los datos dentro de las matrices construidas y se elabora una matriz de cruce de vínculos sociohistóricos y de encuadre teórico de las comunidades. 
ReVista UniversidAd EN DiÁlogo • Vol. 8, N. ${ }^{\circ}$ 2, Julio-Diciembre, 2018, pp. 99-124

ISSN 2215-2849 • EISSN: 2215-4752

DOI: http://dx.doi.org/10.15359/udre.8-2.7

3. Con la información recolectada, sistematizada y analizada se planifican y ejecutan talleres de formación de saberes conceptuales, procedimentales y actitudinales vinculados con la organización social y la conformación de redes con diferentes grupos. Se realiza un acompañamiento pedagógico mediante acciones de trabajo conjunto entre extensionistas, estudiantes y la comunidad.

4. Se sistematiza y analiza la información recolectada y se reflexiona a partir de instrumentos realizados.

\section{Resultados}

Algunos de los resultados que se han identificado desde la investigación y extensión enmarcan una serie de logros, los cuales se generan tanto en el grupo académico y los y las estudiantes extensionistas como en las comunidades en que se están realizando acciones educativas.

\section{Factores que inhiben y potencian el desarrollo social de las comunidades de Santa Rita y Los Chiles de Alajuela, desde una lógica de entrecruzamiento sociohistórico}

El diagnóstico realizado permitió identificar los factores que inhiben y que potencian a cada comunidad, los cuales son la base para el desarrollo de las acciones ejecutadas en cada una de ellas. Este diagnóstico es producto de observaciones y visitas de campo, así como entrevistas a distintos actores comunales, encuentros informales y trabajo con varios grupos comunales. Entre los factores identificados se puntualizan los siguientes: económicos, sociales, políticos y organizacionales, culturales, servicios e ingreso a la comunidad, educativos. El parámetro teórico para comprender las dinámicas que se explican viene dado por el entrecruzamiento histórico, los factores descritos reciben la fuerza de dos grandes procesos mundiales aparentemente exógenos: globalización y capitalismo mundial (Acuña, 2014).

En las comunidades fronterizas estudiadas, la estructura productiva eminentemente campesina, sumamente orientada a la pequeña propiedad, es violentada por la impronta de empresas nacionales y transnacionales de la fruta, lo que obliga a muchos/as pequeños/as productores/as a alquilar sus tierras a dichas compañías. 
Además, el ingreso de braceros proletarios campesinos desde Nicaragua altera la dinámica comunitaria, su llegada, promovida por las empresas fruteras, representa un abaratamiento de la mano de obra y genera una alta tasa de embarazos, lo que resulta en una gran cantidad de madres jefas de familia, embarazo adolescente y frustración sobre todo en la población femenina.

Para las cuatro comunidades, la carencia de un currículum educativo contextualizado en escuelas y colegios que potencie nuevas formas organizacionales limita las habilidades blandas de las poblaciones. El vacío de espacios de convivencia social y la oferta limitada de grupos comunales generan profundas disputas en los pobladores en la búsqueda de la representación y reconocimiento personal.

La oferta cultural es mínima, y no se profundiza el trabajo de rescate identitario, lo que es aprovechado por una invasión de los medios de comunicación, ante todo redes sociales y televisión. Ante el vacío institucional y recreativo, la llegada de iglesias fundamentalistas aniquila la ideología popular, altera la herencia histórica y copta la organización popular.

En algunos casos, como en la iglesia metodista en San Gerardo (frontera norte), se pueden lograr puentes comunicativos importantes y de mayor respeto identitario. En el caso de la iglesia católica, sobre todo en Punta Cortés y el Cachito, por ser comunidades atendidas por franciscanos, tienden a privar elementos de la teología de la liberación, los cuales procuran el respeto y rescate de la fe popular. El caso de la llegada, consolidación y fortalecimiento de las iglesias fundamentalistas de corte pentecostal es sumamente dramático, sobre todo en el caso de Santa Rita, en la periferia del cantón central de Alajuela.

La ideología popular es socavada, y las formas organizacionales populares existentes son asumidas por líderes espirituales que, con base en una lógica viejo testamentaria y paulista, condenan ante todo a la mujer a situaciones de sumisión, obediencia y aceptación de un patriarcado heredado históricamente y ahora reforzado por textos bíblicos considerados inapelables y sagrados.

Toda manifestación organizacional, artística o educativa que violente los principios fundamentalistas es considerada como obra de poderes demoniacos y sujeta a la vigilancia y al recelo ante todo de hombres que han asumido el rol de pastores, diáconos y son legitimados por profetas y apóstoles que operan desde gran parte de iglesias evangélicas a nivel nacional 
ReVista UniversidAd EN DiÁlogo • Vol. 8, N. ${ }^{\circ}$ 2, Julio-Diciembre, 2018, pp. 99-124

ISSN 2215-2849 • EISSN: 2215-4752

DOI: http://dx.doi.org/10.15359/udre.8-2.7

y transnacional. No obstante, sectores de mayor edad, con pasados rurales, mantienen ideas más liberales, experiencias de vida y costumbres heredadas de un catolicismo soterrado en sus ideologías que los torna más críticos y apáticos ante el discurso fundamentalista, lo que ha generado una red sumamente fuerte de personas adultas mayores.

\section{Esbozos de propuestas metodológicas para el logro de aprendizajes organizacionales que propicien el desarrollo de redes comunales socioeducativas en las comunidades de Santa Rita y Los Chiles en la provincia de Alajuela}

La tabla 2 muestra algunos de los resultados que se vinculan con los objetivos presentados en este artículo. Se trata de una rápida mirada a las acciones creadas con y desde las comunidades; no obstante, es necesario profundizar más en escritos posteriores.

En todas las comunidades se desarrolla un trabajo sociopedagógico que retoma aquellos elementos que caracterizan todos los encuentros con las personas de las comunidades, entre ellos: la espiritualidad, comensalidad, diálogo, actividad lúdica, experiencias de aprendizaje, respeto, cortesía, entre otros. Estos principios, como se dijo en el marco teórico, retoman la posición de Florez (2005) para la pedagogía como una ciencia unificada de la educación, que mira siempre un nivel conceptual de formación.

Estas acciones que se desarrollan en las comunidades con las redes conformadas permiten diferentes experiencias, vivencias y aprendizajes que le dan sentido y significado a la presencia del proyecto y a la presencia de las personas que son parte de estas comunidades. Como se señaló en el marco teórico, el aprendizaje en red está ligado a la dinámica de la vida, porque la vida misma es una red.

Es importante que las actividades de ocio y recreación hayan resultado fundamentales en todas las comunidades para la activación del trabajo pedagógico focalizado en las habilidades organizacionales. Como se señaló en el marco teórico, la lúdica es un principio pedagógico fundamental en la acción, tal como lo señala Florez (2005) al abordar el principio de la lúdica en toda propuesta pedagógica. En cuanto al ocio y a la recreación -y desde un enfoque pedagógico que se desarrolla en las comunidades como una forma de compartir y redimensionar todas sus situaciones-, a continuación, se describen una serie de actividades desarrolladas en estos espacios: 
Revista Universidad en Diálogo • Vol. 8, N. ํㄹ Julio-Diciembre, 2018, pp.99-124

ISSN 2215-2849 • EISSN: 2215-4752

DOI: http://dx.doi.org/10.15359/udre.8-2.7

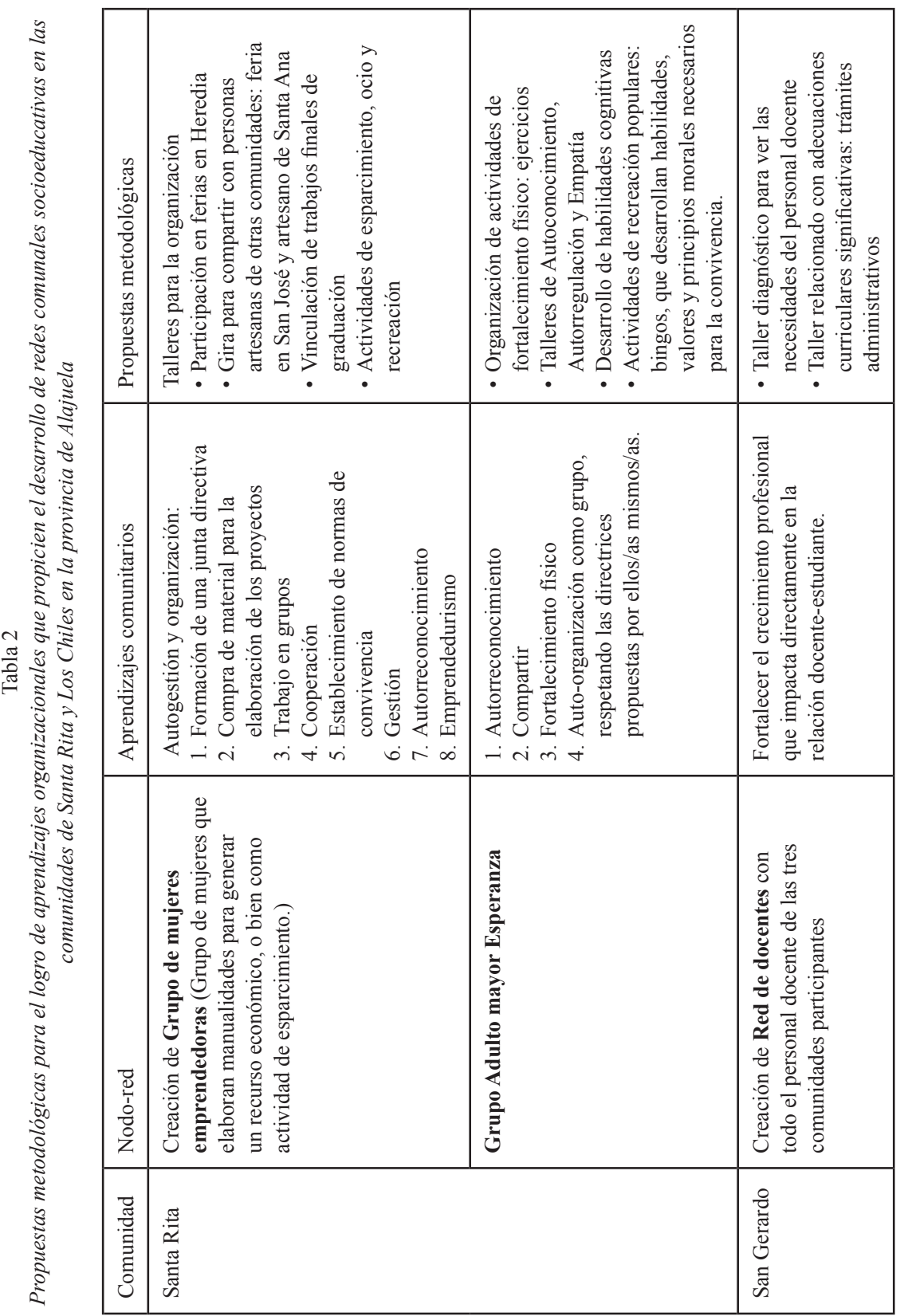




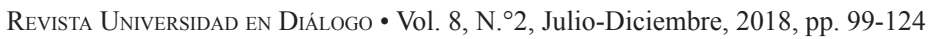

ISSN 2215-2849 • EISSN: 2215-4752

DOI: http://dx.doi.org/10.15359/udre.8-2.7

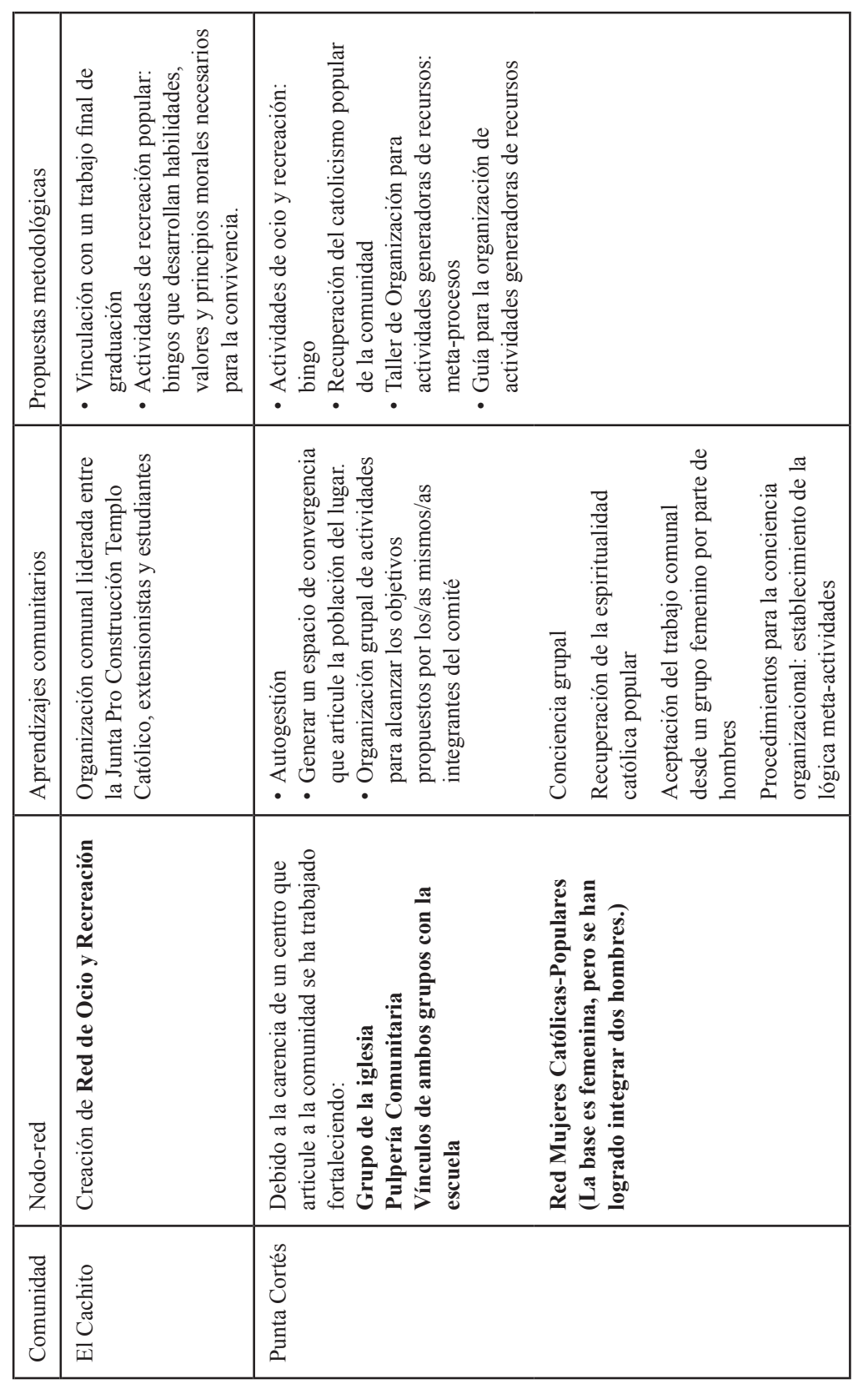

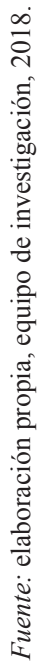


Bingo: En el cual se genera aprendizaje como recurso para la auto-organización; es un espacio que permite el encuentro, el diálogo y la recreación.

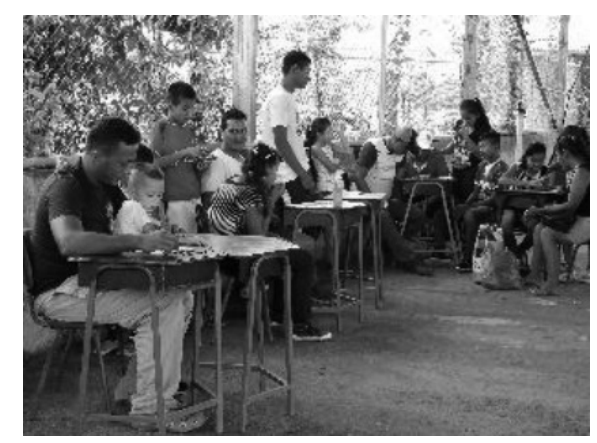

Tardes de recuerdo: Una experiencia mágica que les permite recordar historias a través de la conversación con el otro, de lo espiritual y de aprendizajes mutuos.
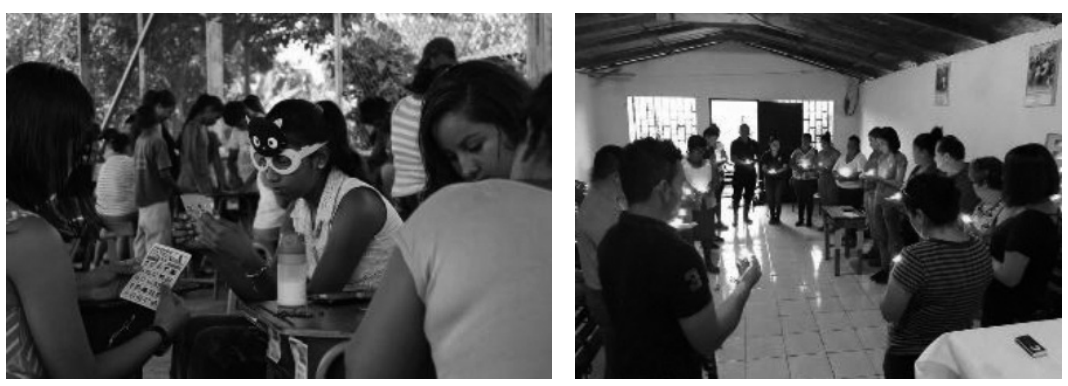

Tertulias grupales: Basadas en temas de interés, acompañadas de un cafecito.

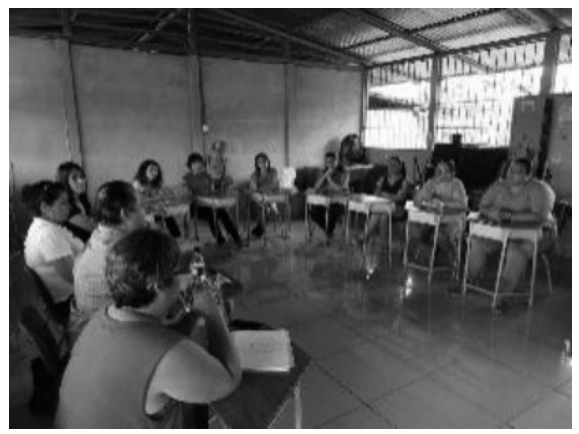


Revista Universidad en DiÁlogo • Vol. 8, N. ${ }^{\circ} 2$, Julio-Diciembre, 2018, pp. 99-124

ISSN 2215-2849 • EISSN: 2215-4752

DOI: http://dx.doi.org/10.15359/udre.8-2.7

Terapia física y movimiento recreativo: Para promover el cuidado de la salud cognitiva, física y emocional por medio de actividades físicas para mejorar la calidad de vida
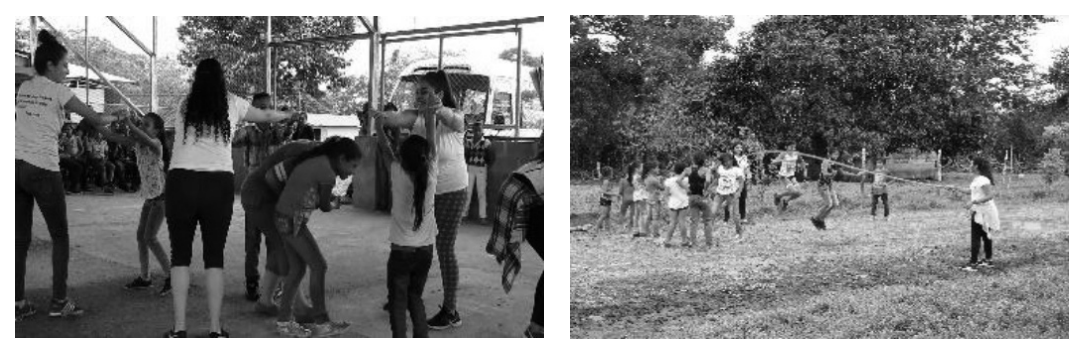

Talleres con población infantil y otras personas de la comunidad: Se imparten diferentes áreas, tales como arte, movimiento, higiene, entre otras. Todos los encuentros realizados consistieron en la co-creación de estrategias que incentiven la promoción del ocio y la recreación en las personas de la comunidad.
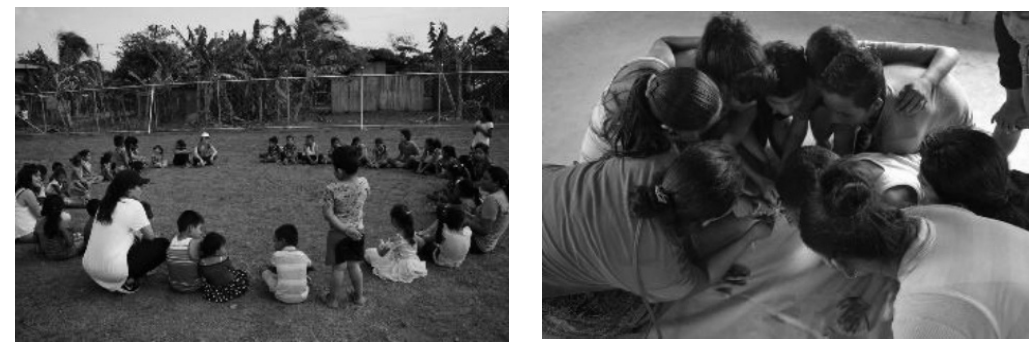

\section{Conclusiones}

Para valorar las estructuras organizacionales y de convivencia social y para construir aprendizajes organizacionales para el desarrollo de redes comunales socioeducativas en las comunidades, se ha partido de la búsqueda y consolidación de los grupos o formas propias de organización comunitaria ya creadas. Los mismos deben hacerse visibles para los y las participantes mediante el diálogo y el autorreconocimiento de la existencia de dichos grupos. La forma de activar los protogrupos nodales que conforman las redes comunales parte de metas a corto plazo, involucrando la acción y los espacios de ocio-recreativo y de encuentro comunal. 
Las poblaciones presentan múltiples dinámicas que tienden a inhibir su capacidad y deseo organizacional, algunas vinculadas con el uso de la tierra, otras con currículos educativos alejados de las lógicas ideológicas y productivas de las comunidades. Además, en el caso de las comunidades fronterizas, los caminos y vías de comunicación con centros de población mayores hacen muy difícil la movilización de recursos y de personas. La impronta de las iglesias protestantes, sobre todo en San Gerardo y muy marcadamente en Santa Rita, generan un total control de la organización popular, coptando todo tipo de acciones y sometiendo ante todo a la mujer a patrones patriarcales que las hunden en la sumisión y la violación de sus derechos. La dinámica de las empresas fruteras que operan desde la lógica de encontrar terrenos a bajo precio (mediante el alquiler de los mismos) y la estrategia de permitir el flujo migratorio ilegal para asegurarse una abundante oferta de mano de obra barata pero volátil hace que exista poco arraigo e interés en la organización comunal por parte de dicho sector.

Por otra parte, la creación de acciones educativas sociales parte ante todo del respeto y cocreación de líneas de trabajo, las cuales se fundamentan en los siguientes aspectos:

- La relación entre la universidad y la sociedad tiene como fin orientar adecuadamente los esfuerzos colectivos desde una mirada más inclusiva.

- El crear y desarrollar redes comunales en comunidades en riesgo social y cercanas al cordón fronterizo norte ha sido todo un reto y un aprendizaje tanto para el grupo de investigación como para las comunidades y estudiantes participantes, ya que en la investigación y en la extensión se debe mantener una estrecha relación que implica la creación y desarrollo de espacios comunales que garanticen el respeto a la identidad cultural para entender las dinámicas comunales y de organización de cada lugar, además de la formación en valores y la formación profesional de docentes y estudiando con un perfil amplio comprometidos en el ámbito social y profesional. Las ideas de construcción conjunta con los actores sociales son propias de una investigación cualitativa.

- La lógica etnográfica y de entrecruzamiento entre las comunidades le ha permitido al grupo de investigación-extensión apropiarse de esas construcciones comunales y darles un sentido pedagógico sin violentar sus procesos sociales. 
- El proyecto aporta desde su bagaje teórico y metodológico y de forma integral propicia la capacidad de mirar e interactuar con todo tipo de diversidad y la capacidad de organización, y respecto a la dinámica de las comunidades, la facilidad de aportar a estas la promoción de acciones pedagógicas no formales y formales centradas en el ocio recreativo y la recreación.

- Los procesos educativos, los espacios para el ocio y la recreación en las comunidades de Santa Rita y Los Chiles son muy limitados, por tal motivo la concretización de espacios pedagógicos ha permitido aprendizajes, disfrute, lograr mecanismos más efectivos para la organización, el diálogo y mejorar la calidad de vida en su tiempo libre.

- Las experiencias que el proyecto activa por medio de la extensión como una forma de transformar la sociedad, para lo cual se hace imprescindible el reconocimiento de la comunidad como un elemento fundamental para la formación humana colectiva. Este reconocimiento se logra estableciendo una relación estrecha con la comunidad y compartiendo no solo en la formalidad de la investigación, sino también en actividades ocio-recreativas donde espacios para el aprendizaje surgen de manera natural. Se hace énfasis en una educación de aprendizajes compartidos.

- A través de la ejecución del proyecto se identifican algunos principios que pueden retomarse en el planteamiento de extensión universitaria como equidad, responsabilidad, pertinencia social y autonomía institucional, los cuales responden a una universidad dinámica que desarrolle procesos de capacitación, asegure la igualdad, la participación común, el progreso económico, la reflexión y análisis, la tolerancia y la justicia social.

- La estrecha relación entre la universidad y la sociedad influye y transforma de manera positiva el entorno; por tanto, ayuda a construir una sociedad con determinadas características e incentiva la participación comunitaria.

- Buscar la solución de problemas críticos que aquejan a la sociedad es un deber de la universidad a través de la investigación, docencia 
y extensión, esto se traduce en cooperación entre proyectos interdisciplinarios, entre unidades y facultades, con una visión integral tanto en su acción sustantiva como en el financiamiento de recursos para los mismos. La integración de estudiantes universitarios/as mediante sus propuestas de trabajos finales de graduación y en la modalidad de estudiante asistente se convierte en un elemento esencial para la ejecución de los proyectos.

- Legitimar la pertinencia social de la universidad es tener los objetivos claros en cuanto a la relación con la sociedad, en función de las necesidades y demandas sociales.

- El papel de la persona docente en la producción del conocimiento en los procesos de extensión universitaria debe orientar permanentemente la relación entre el saber académico y el saber popular y promover formas de asociación, líneas de investigación e innovar planes de estudio.

\section{Referencias}

Acuña, V. (2014). Centroamérica: filibusteros, Estados, Imperios y memorias. San José, Costa Rica: Editorial Costa Rica.

Bedma, M. y Añaños, F. (2007). Introducción a la pedagogía social/ educación social. Malaga, España: Grupo Editorial Universitario.

Barone, S. (2009). Las estructuras organizacionales: cultura, principios y modelos para la organización. República Dominicana: Editorial Corripio.

Berns, J. y Fitzduff, M. (2007). Enfoques complementarios del trabajo de convivencia ¿Qué es la convivencia y por qué adoptar un enfoque complementario? Estados Unidos de América: Alan B. Slifka Foundation.

Capra, F. (2017). La trama de la vida. Una nueva perspectiva de los sistemas vivos. España: Editorial Anagrama.

Flórez. R. (2005). Pedagogía del conocimiento ( $2^{\mathrm{a}}$ ed.). Bogotá, D.C., Colombia: McGraw-Hill Interamericana. 
ReVista UniversidAd EN DiÁlogo • Vol. 8, N. ${ }^{\circ}$ 2, Julio-Diciembre, 2018, pp. 99-124

ISSN 2215-2849 • EISSN: 2215-4752

DOI: http://dx.doi.org/10.15359/udre.8-2.7

Flórez, R. y Tobón, A. (2001). Investigación Educativa y Pedagógica. Bogotá, Colombia:

Mc Graw Hill.

Freire, P. (1985). Pedagogía del oprimido. México: Siglo XXI Editores.

Gutiérrez, F. (2006). Doctorado de la III Cultura. En busca del sentido. Costa Rica: Editorial Nuestra Tierra.

Morin, E. (2014). La vía para el futuro de la humanidad. España: Editorial Planeta. 